\title{
Retos de integración en el EEES: Máster Universi- tario en Profesor de Educación Secundaria Obligatoria y Bachillerato, Formación Profesional y enseñanza de idiomas y los TFM
}

\author{
Javier RODRÍGUEZ TORRES \\ Universidad de Castilla - La Mancha \\ javier.rtorres@uclm.es
}

Recibido: 09/07/2012

Aceptado: 28/09/2012

\begin{abstract}
Resumen
La integración en el EEES supone para los docentes cambios profundos. La primera diferencia que se encuentra al comparar los antiguos programas del CAP y el Máster es que, si bien en aquellos su núcleo organizador eran los contenidos, encontramos ahora que el núcleo organizador son las competencias. Al comenzar la IV edición, adquiere una especial dimensión que los docentes evaluemos nuestra propia actuación, obtengamos información del proceso y de los resultados y reflexionemos en un proceso de mejora continua. Queremos en este trabajo exponer los resultados de un estudio que tiene por objeto determinar en qué medida los alumnos y alumnas: a) valoran la relevancia de las competencias explicitadas en las Guías docentes de las asignaturas; y b) en qué medida las han desarrollado a través de sus TFM

Palabras clave: Máster Secundaria, TFM, EEES, competencias, CAP (Certificado de Aptitud Pedagógica)

\section{Challenges of Integration in the EHEA: University Master in Teacher of Secondary Obligatory Education and Baccalaureate, Vocational Training and language Teaching and the WEM}

\begin{abstract}
The integration in the European Higher Education Area (EHEA) involves great changes to the teachers. The first difference that is found comparing the training programs CAP* and Master is that in those ones, the organizing core were the contents, but we find that the competences are now the organizing core. Beginning the IV edition, it acquires a special dimension that the teachers are able to evaluate our own action, we could obtain/get information from the process and its results, and we should think about a constant/continuous improvement process. In this work we want to expound the research results (of a study) which its aim is to determine in what extent the students are able to: a) value the relevancy of the competences in the Teachers' Guides of the subjects; and b) value in what extent they have developed them through their Work End of Master (WEM)

Keywords: Secondary Education Master, WEM, EHEA, ESHE, competences. Pedagogical Aptitude Certificate

\section{Referencia normalizada}

RODRÍGUEZ TORRES, Javier (2012): "Retos de integración en el EEES: Máster Universitario en Profesor de Educación Secundaria Obligatoria y Bachillerato, Formación Profesional y enseñanza de idiomas y los TFM". Estudios sobre el mensaje periodístico. Vol. 18, núm. especial noviembre, págs.: 791-799. Madrid, Servicio de Publicaciones de la Universidad Complutense.

Sumario: 1. Introducción; 1.1. Organización del Máster; 1.2. TFM en la Universidad de Castilla - La Mancha; 1.3. Revisión literaria. 2. Metodología: instrumentos, participantes y procedimiento. 3. Desarrollo. 4. Conclusiones: discusión, limitaciones y prospectiva. 5. Referencias bibliográficas.
\end{abstract}




\section{Introducción}

La formación pedagógica y didáctica del profesorado de Educación Secundaria antes de la implantación del EEES se reducía al curso para la obtención del Certificado de Aptitud Pedagógica (CAP), sobre el que la mayoría del profesorado opinaba sobre su baja eficacia ${ }^{1}$. Es decir, es CAP es percibido por la mayoría del profesorado como un requisito burocrático para acceder a las oposiciones y/o al trabajo y no como una formación imprescindible para ser un profesional de la educación competente.

Desde la implantación de la LOGSE se han realizado intentos legislativos por modificar el CAP mediante distintos RRDD, que fueron sufriendo retrasos, congelaciones o no llegan a ponerse en práctica: Real Decreto establecía el Curso de Cualificación Pedagógica (CCP) (1995), Propuesta de curso de formación pedagógica del profesorado, que plantearía una situación intermedia entre el antiguo CAP y el CCP (MECD, 2001), Real Decreto 118/2004, de 23 de enero, por el que se regula el título de Especialización Didáctica.

Así pasarán unos años más hasta el Real Decreto 1393/2007 por el que se establece la ordenación de las enseñanzas universitarias oficiales, los nuevos planes de estudios tendrán que contemplar en su diseño tanto las competencias que figuren el Marco Europeo de Cualificaciones para la Educación Superior como las competencias que establezca el Marco Español de Cualificaciones para la Educación Superior (Real Decreto 1027/2011, de 15 de julio). Por otro lado, todos los títulos adaptados al Espacio Europeo de Educación Superior (EEES) deben cumplir con los estándares europeos de garantía de calidad (ENQA, 2005) que exigen procedimientos de evaluación fiables, válidos y transparentes. Asimismo, el Real Decreto 1393/2007 establece la obligatoriedad de finalizar las enseñanzas oficiales de Grado y de Máster con la elaboración y defensa de un trabajo.

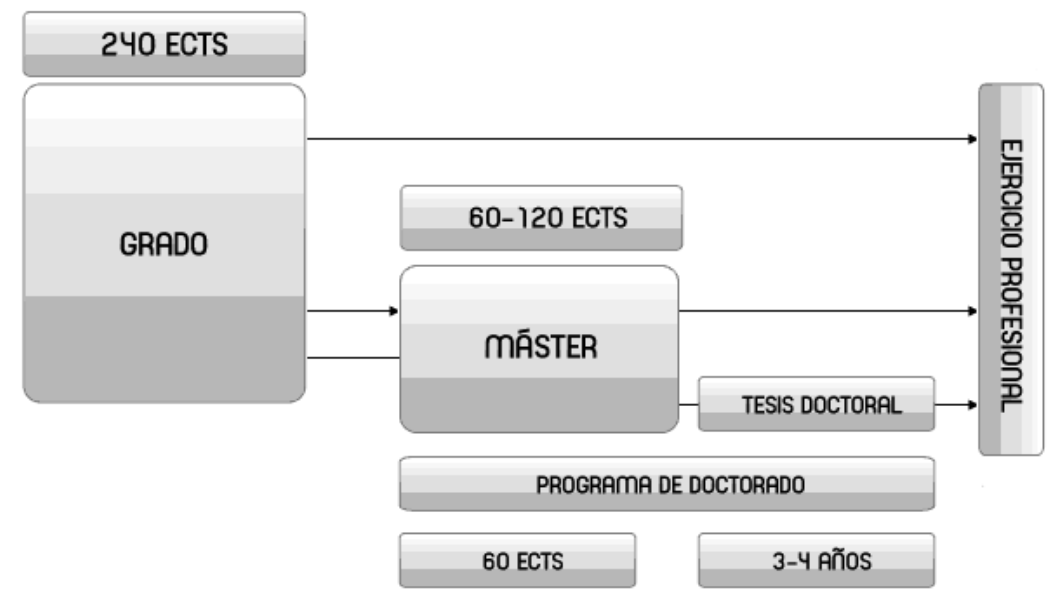

${ }^{1}$ En encuesta del MEC, 1986: 102 el 73.1 \% de BUP y 60,4 \% de FP su opinión general era: «No me ha servido para casi nada» 


\subsection{Organización del Máster ${ }^{2}$}

Para ejercer la docencia en la Educación Secundara Obligatoria, el Bachillerato, la Formación Profesional y la Enseñanza de Idiomas (MPES), será necesario estar en posesión de un título oficial de máster que acredite la formación pedagógica y didáctica de acuerdo con lo exigido por los artículos 94, 95 y 97 de la LOE 2/2006, de 3 de mayo, de Educación. La ORDEN ECI/3858/2007, de 27 de diciembre, por la que se establecen los requisitos para la verificación de los títulos universitarios oficiales que habiliten para el ejercicio de las profesiones de Profesor de Educación Secundaria Obligatoria y Bachillerato, Formación Profesional y Enseñanzas de Idiomas establece los requisitos para la verificación del Título:

12 créditos ECTS a un Módulo Genérico de contenido claramente pedagógico, que incluirá: Aprendizaje y desarrollo de la personalidad (5créditos), Procesos y contextos educativos (5 créditos), y Sociedad, familia y educación (2 créditos).

24 créditos a un Módulo Específico orientados al:

- Aprendizaje y enseñanza de las materias correspondientes: didácticas específicas de cada materia

- Complementos para la formación disciplinar: pretende paliar la especialización de los grados en una sola materia de las que componen algunas áreas que el profesorado debe impartir en Educación Secundaria.

- Innovación docente e iniciación a la investigación educativa: pretende conseguir el necesario avance continuo en la mejora de los procesos de enseñanza y aprendizaje.

- Formación práctica. Prácticum y TFM: La Orden ECI/3858/2007 establece que se dedicarán al prácticum (18 créditos), y la realización del Trabajo de Fin de Máster (6 créditos).

\subsection{TFM en la Universidad de Castilla - La Mancha}

Tal como consta en el Real Decreto 1393/2007, el proceso concluye con la elaboración y defensa de un trabajo fin de Máster que, en el caso del Máster de Secundaria, es de 6 créditos. Desde la Orden ECI/3858/2007, apartado 5 del anexo se establece que el Prácticum se realizará en colaboración con las instituciones educativas establecidas mediante convenios entre Universidades y Administraciones Educativas.

La Normativa de la UCLM a este respecto señala que el trabajo fin de Máster ha de estar bajo la supervisión del tutor de prácticas en el IES y del tutor de la Universidad, y orientarse a la aplicación de las competencias generales del título, de forma que se permita evaluar los conocimientos y capacidades adquiridos por el estudiante en las distintas materias del Máster.

Desde el inicio en la UCLM, el objetivo en relación con esta materia ha sido reforzar el carácter particular de este trabajo, como corresponde a unas prácticas profesionales universitarias (Manzanares, 2010a). Tratando de evitar la consideración de una simple memoria recopilatoria de lo aprendido durante todo el Máster o, básica-

${ }^{2}$ Las referencias concretas a créditos ECTS se corresponden con la organización y distribución en la UCLM 
mente, durante las prácticas. Se ha insistido en que se trata de un trabajo original, fundamentado teóricamente y con una propuesta docente ajustada a la normativa vigente, para su consecución se propone que dicho trabajo tenga tres posibles vías: la Programación Docente, la Innovación Docente y la Investigación Educativa, de tal forma que la realización del TFM durante el Prácticum hará que la colaboración a la vez que necesaria sea beneficiosa para los dos colectivos de profesores. El papel del tutor o tutora del centro de prácticas, aquí, es esencial. Tal y como se recoge en la Guía para tutores de la UCLM (Manzanares, 2010b)

Cabe destacar es que el TFM en la UCLM se considera un documento reflexivo donde se solicita al estudiante deje constancia del nivel de desarrollo adquirido de la competencia para enseñar dentro de la especialidad docente o para realizar las funciones de orientación. Para ello la labor del tutor o tutora del centro de prácticas es fundamental. En el trabajo con el tutor, el estudiante ha de decidir qué actividades o tareas permiten un desarrollo de las competencias del título y, también, qué otras le colocan en situación de trabajar las competencias de los alumnos de los grupos que el tutor ha estimado conveniente. De otra parte, los tutores de universidad, orientan que refleje el nivel de logro de las competencias docentes desarrolladas por el estudiante conforme a su formación inicial y al aprovechamiento que ha realizado del Máster.

\subsection{Revisión literaria}

Desde el acuerdo de Bolonia (1999), nuestra legislación y las distintas universidades del país han implementado el MPES que tiene como fin la formación psicopedagógica rigurosa, acorde con las necesidades del sistema educativo actual y con las características de un aprendizaje profesional de calidad. Desde la Universidad se pretende lograr una formación inicial, que se convierta en un modelo de referencia para la formación universitaria de los futuros profesionales de la enseñanza secundaria, que se fundamenta tanto en la previa experiencia en el desarrollo del CAP, como en las directrices del Ministerio de Educación enmarcadas en las orientaciones del EEES.

Para tener una visión general, remitimos al documento de la ANECA sobre la configuración del Master en las Universidades del país ${ }^{3}$.

Para la metodología empleada en nuestro trabajo y por potenciar la reflexión sobre las experiencias que tienen lugar en la propia práctica constituyendo la materia prima de nuestro estudio, hemos optado por la etnografía y el diario de campo. Esta orientación etnográfica-narrativa ha sido trabajada por diversos autores para la investigación educativa (Zabalza, 2004; García 2000; Anguera, 1988). El diario de los profesores configura una de los apartados más relevantes en este contexto de investigación etnográfico-educativa (Porlán, 2008; Martínez y Sauleda, 2002). La funcionalidad evaluativa -autoevaluativa- del diario de campo, su potencial reflexivo sobre la acción en los procesos de enseñanza aprendizaje y su instrumentalización para retroalimentar la práctica educativa ha sido destacada por diversos autores (Cano, 2009; Kemmis, 1999), que coinciden en señalar el carácter "pedagógico" de esta herramienta reflexiva en aras a su positiva incidencia en la formación de profesionales del sistema educativo.

${ }^{3}$ http://es.scribd.com/doc/88012428/Informe-Master-Secundaria-ANECA 


\section{Metodología: instrumentos, participantes y procedimiento}

Partiendo del marco teórico, la revisión bibliográfica y legislativa, nuestras Guías docentes, los datos obtenidos en las tres promociones del Master como profesor de Materia de Módulo Común, director de TFM y miembro de distintos Tribunales de TFM, decidimos investigar sobre:

- la relevancia de las competencias explicitadas en las Guías docentes de las asignaturas

- en qué medida las han desarrollado a través de sus TFM y

- efectuar un ejercicio evaluación formadora que, más allá de cualquier otra evaluación institucional externa, debe favorecer la secuencia de mejora de la calidad docente

Otros instrumentos, no menos valiosos para nosotros, han sido los generados en el propio proceso de aprendizaje de los alumnos y alumnas a los que hemos tenido acceso, a saber:

- Materiales generados: trabajos individuales y grupales (2 grupos de Toledo de 3 promociones, alrededor de unos 350 alumnos)

- TFM de distintas especialidades (30 de las especialidades de Lengua y Literatura, Educación Física y Orientación Educativa)

- Cuestionarios

- Análisis de competencias

El enfoque de investigación por el que nos decantamos tiene un significado social, ideológico y político, pero la concreción práctica y la dirección que adoptamos requieren de unos principios de procedimiento que conviertan las actividades y estrategias de investigación en un proceso de formación compartido y recíproco entre investigador y los futuros docentes.

Para nosotros ha sido de gran ayuda en el momento de diseñar y desarrollar esta investigación, así como para comprender que ésta debía de convertirse en un proceso de saber-poder democrático. Reflexionar sobre los valores educativos que subyacen en cada enfoque y los intereses a los que sirve cada uno de ellos. Para Elliott la «investigación sobre la educación» estaría al servicio de intereses ajenos a la realidad educativa y se pone al servicio de los investigadores y no de los docentes y el alumnado. Estaríamos ante un tipo de investigación individualista que se basa en la distinción clara y jerárquica del trabajo de docentes e investigadores, por lo que no implica ningún cambio ni mejora para los profesores y alumnos. Por el contrario, la «investigación educativa» está al servicio de la práctica educativa, en tanto sirve para dar respuesta a los intereses de docentes y alumnos. Es particularmente importante tener en cuenta que en este enfoque de investigación los valores educativos son una finalidad que debe concretarse en el mismo proceso de investigación.

La investigación así planteada se convierte en un proceso de aprendizaje y perfeccionamiento colectivo al estar conectada con la práctica misma. Lo que se traduce en este trabajo en que todos los participantes en el proceso conozcan y comprendan las dificultades, problemas y aciertos que el uso de estos recursos genera en situacio- 
nes educativas concretas y, en consecuencia, las mejoren desde la realidad cotidiana de sus centros.

El diseño se conformó por varias unidades de análisis que emergían como escenarios permitiéndonos conocer y analizar cómo y en qué afecta la realización del Máster a su adquisición de competencias cuando éstos realizan sus actividades de formación y cuando las utilizan para desarrollar las actividades escolares en los centros donde realizan el Prácticum. Se recogen las acciones más significativas desarrolladas durante nuestro trabajo de campo a través de la observación participante, entrevistas, grupos de discusión, análisis documental y distintos cuestionarios, para acercarnos a nuestro objeto de estudio y el carácter singular de los estudiantes implicados.

\section{Desarrollo}

Disponemos de datos para hacer un análisis, sin embargo, éste es necesariamente provisional y parcial, al disponer sólo de datos directos de nuestra Universidad.

Durante el primer año el Máster parecía no satisfacer las expectativas de nadie por completo, los problemas de gestión y coordinación, las añoranzas y simplicidad del CAP con respecto a la docencia y Prácticum. Todo lo anterior motivó que desde distintas estancias se criticase, escondiendo, desde nuestra perspectiva, un nuevo ataque a la LOE y a la pujante necesidad de inclusión de la pedagogía en la formación inicial de los docentes. El trabajo bien hecho por los docentes y estudiantes, la implicación progresiva de los centros y el impulso desde la Institución, nos sitúan en un panorama muy distinto al del primer curso.

Con respecto a la matrícula y finalización de estudios los datos que tenemos de nuestra Universidad son los siguientes:

\begin{tabular}{|l|c|c|c|}
\hline & CURSO 2009-10 & CURSO 2010-11 & CURS0 2011-12 \\
\hline $\mathrm{N}^{\mathrm{o}}$ ALUMNOS & $\mathbf{5 2 3}$ & $\mathbf{3 9 4}$ & $\mathbf{3 9 9}$ \\
\hline ESPECIALIDADES & $\mathbf{1 5}$ & $\mathbf{1 5}$ & $\mathbf{1 4}$ \\
\hline FINALIZAN & $\mathbf{5 0 1}$ & $\mathbf{3 1 7}$ & $\mathbf{3 7 7}$ \\
\hline
\end{tabular}

Mediante nuestra experiencia con los TFM, reuniones de trabajo con colegas comprobamos que los estudiantes hacen, también impulsado por una interpretación de la propia Universidad y Tutores de los TFM, de una variada modalidad en los trabajos:

- el TFM como memoria del Prácticum;

- el TFM como una programación didáctica;

- en algunas, las menos de las veces, el TFM como la realización de un trabajo original en el que se elabore una propuesta educativa a partir de los contenidos del módulo genérico, del módulo específico, del Prácticum o de una combinación de todos.

Desde nuestro parecer un aspecto clave en el Master es la investigación educativa. Si bien es cierto, que la duración del Prácticum es corta y que muchos de los estudiantes carecen de experiencia docente, la investigación educativa debe ir ligada a la innovación. Los aprendizajes del modulo específico Innovación docente e iniciación 
a la investigación educativa junto con las bases que proporciona el Modulo común y el periodo de prácticas; constituyen unos buenos soportes para adquirir las competencias exigibles para otorgar el título, según la Orden ECI 3858/2007, de 27 de diciembre.

La realidad que encontramos en los TFM, refleja una necesidad de mejorar este elemento clave de calidad, los estudiantes reflejan aproximaciones a la innovación con las siguientes tipologías:

- Muy vinculados a las TIC. Relacionan el uso de los medios con la innovación y como ya sabemos por otros trabajos, esta no siempre es una relación bi - unidireccional

- Poco fundamentados, basados en intuiciones "cosas que no se hacen"

- Alejados del continuo curricular, se olvidan los currícula como fuente de innovación y cambio.

- Sujetas a contradicciones metodológicas.

- Confusión constante entre cambio e innovación.

Ante los resultados observados, en algunas sesiones de trabajo interdisciplinares hemos planteado la necesidad de la implicación pedagógica en el módulo de innovación docente, que actualmente en nuestra Universidad se asigna a las Didácticas específicas.

Una de las razones más contundente, desde nuestro punto de vista, es que la formación pedagógica, investigación e innovación educativa no debiera otorgarse completamente a quienes no conocen, ni tienen experiencia docente en el nivel para el que van a preparar a los estudiantes. La investigación educativa e innovación debería estar basada en la experiencia real de los profesores, en su trayectoria de investigación educativa e innovación, de no ser de esta forma, se corre el riesgo de adoctrinamiento disciplinar dogmático, con clichés, que poco van a aportar al futuro docente con la incertidumbres del trabajo cotidiano.

De nuestro contacto con los estudiantes y la guía en su aprendizaje en los TFM hemos extraído aspectos valiosos que queremos reflejar en este trabajo:

- Iniciamos este camino en el Master con una serie de supuestos que hemos corroborado como erróneos y que no siempre se corresponden con las dificultades presentadas y expuestas por ellos:

- Formación necesaria para desarrollar un trabajo universitario: fuentes de información búsqueda, selección y expresión correcta, bibliografía...

- Formación científica suficiente en la especialidad. Desde la especialidad de Orientación, la más trabajada en nuestro caso en el módulo específico, se encuentran fallas que hay que solventar, mejorando aspectos fundamentales de su formación científica. Esta situación nos ha sido relatada por docentes de otras especialidades que tienen que mejorar su formación científica en sus respectivas áreas de conocimiento.

- Manifiestan las dificultades de acercamiento al currículum de las áreas, algo abstracto y poco relacionado con su disciplina, en principio. Será en el Prácticum donde empiecen a atisbar la complejidad de las relaciones. No en vano, 
alguno nos manifiesta, la necesidad de un breve periodo de Prácticum antes de abordar el Modulo II de Didácticas específicas.

\section{Conclusiones: discusión, limitaciones y prospectiva}

En los programas formación inicial del profesorado, tanto los Grados como el Master, sus Trabajos de Fin de Grado y Master, tienen un papel fundamental para asegurar la integridad de las cualificaciones que se ofrecen a los alumnos y supone un paso importante en la mejora de la formación del profesorado. Esta circunstancia nos exige a todos los implicados el diseño de instrumentos válidos y fiables dentro del modelo de aprendizaje basado en competencias en el que estamos inmersos, para la correcta evaluación de los procesos, una vía, sin duda, de emergencia para nuestro quehacer en el EEES en la Universidad española.

Desde nuestro punto de vista, el TFM al estar incluido en el módulo Prácticum, debe ligarse a la práctica educativa. Así, debería tratarse de un trabajo de innovación y/o investigación educativa a través del cual se evidencie la adquisición por parte del estudiante de muchas de las competencias del Máster. Este TFM debiera complementarse con la inclusión de alguna actividad de programación con elementos de reflexión sobre lo trabajado en el centro educativo durante las prácticas y su relación con lo trabajado en los Módulos del Máster.

El TFM del MPES, requiere de unas directrices propias, diferentes a las exigencias de lo que podría ser un trabajo de fin de master de orientación investigadora, porque la preparación profesional avanzada, orientada a la especialización, académica y profesional de los estudiantes implica claramente su orientación hacia la evaluación de las competencias profesionales, generales y específicas asociadas a la titulación. De otra forma, la elaboración y defensa del TFM tiene que servir de instrumento de síntesis del conjunto de competencias desarrolladas en el Máster a lo largo de los diferentes módulos formativos, siendo recomendable que esté relacionado directamente con la práctica docente de la especialidad correspondiente.

En la UCLM, hemos avanzado en el diseño de protocolos de trabajo que incidan de forma directa en el diseño y desarrollo del Prácticum y, por tanto, en el papel de los tutores. Con este fin se ha elaborado una Guía para tutores para establecer unos mínimos que hagan homologable el trabajo de los estudiantes del Máster, con independencia del centro en el que vayan a realizar sus prácticas docentes y de los tutores asignados. La Guía ofrece información para diseñar tanto el plan de trabajo en las prácticas como el TFM en su estructura y características básicas, así como herramientas para su seguimiento y evaluación.

Lo que algunos consideran "la invasión" de la Pedagogía en la formación inicial del profesorado es ya imparable, los estudiantes del Máster y docentes en ejercicio analizan sus dificultades en el aula más allá de lo disciplinar y empiezan a buscar respuestas en otras fuentes que les ofrecen orientaciones y enfoques diferentes a los tradicionales.

Sin embargo, esta formación pedagógica considerada por muchos como una serie de lucubraciones teóricas sin correspondencia con la realidad, debido a múltiples y complejas causas, nos exige analizar a los pedagogos nuestras propias investigacio- 
nes y la comunicación que de ellas hacemos, nuestra autoestima científica debiera tener idéntico peso a la profesión que ejercemos, sólo de esta forma, la pedagogía comenzaría a apreciarse, conocerse y lo más importante, se iniciaría la inclusión de lo más valioso en las distintas disciplinas en todos los tramos educativos.

\section{Referencias bibliográficas}

ANGUERA ARACIGALA, Ma Teresa (1988): La observación en la escuela. Barcelona, Grao.

CANO GRACÍA, Elena (2009): Nuevas funciones de la evaluación. Serie Aula Permanente. Madrid, Ministerio de Educación.

ELLIOTT, John (1978): “Investigación en el aula: ¿ciencia o sentido común?”, en ELLIOTT, John (1990): La investigación-acción en educación. Madrid, Morata.

GARCÍA JORBÁ, Juan M. (2000): Diarios de campo. Madrid, Anaya.

KEMMIS, Stephen (1999): "La investigación acción y la política de la reflexión”, en: ANGULO RASCO, José Félix; BARQUÍN, RUÍZ Javier; y PÉREZ GÓMEZ, Ángel I.: Desarrollo profesional del docente: politica, investigación y práctica. Madrid, Akal, pp. 95-118

MANZANARES MOYA, Asunción (2010a): "Las prácticas profesionales universitarias", en PAREDES LABRA, Joaquín y DE LA HERRÁN GASCÓN, Agustín: Cómo enseñar en el aula universitaria. Madrid, Pirámide, pp. 133-145.

MANZANARES MOYA, Asunción (2010b): Guía para tutores del Máster en Profesor de Educación Secundaria Obligatoria y Bachillerato, Formación Profesional y Enseñanza de Idiomas de la Universidad de Castilla La Mancha. Ciudad Real, Ademe.

MARTÍNEZ RUIZ, Ma Ángeles y SAULEDA PARÉS, Narciso (2002): Las narrativas de los profesores: una perspectiva situada Alicante. Editorial Club Universitario.

MINISTERIO DE EDUCACIÓN Y CIENCIA (MEC, 1986): Informe sobre la formación permanente del profesorado de enseñanza básica y secundaria. (19831986). Madrid, AMEC/Subdirección General de Perfeccionamiento del Profesorado.

PORLÁN ARIZA, Rafael (2008): El Diario de Clase y el Análisis de la Práctica. Sevilla, Junta de Andalucía, Consejería de Educación y Ciencia.

ZABALZA BERAZA, M. Ángel (2004): Diario de clase. Madrid, Narcea.

\section{Javier RODRÍGUEZ TORRES}

Profesor titular de Didáctica y Organización Escolar

Universidad de Castilla - La Mancha (UCLM, Toledo) 